\title{
Estrategia psicopedagógica en la educación
}

\author{
Psychopedagogical strategy in education
}

Jorge Rodrigo Andrade Albán. ${ }^{1}$, Noemi Andrada Albán. ${ }^{2}$, \& Víctor Peñafiel Gaibor. ${ }^{3}$

Recibido: 10-10-2018 / Revisado: 17-11-2018 /Aceptado: 04-12-2018/ Publicado: 05-01-2019

\begin{abstract}
.
DOI: $\underline{\text { https://doi.org/10.33262/cienciadigital.v3i1.285 }}$

The ludic expressions have not only benefited man in his history, although now it is known that it is a key activity for the formation of man in relation to others, with nature and with himself insofar as it fosters an aesthetic equilibrium and morality between his interiority and the environment with which he interacts. The objective of this study is to develop an educational proposal that promotes the development of positive attitudes of children both in the classroom and beyond, through the implementation of cooperative games that directly promote the school environment of children of Initial Education. In this line it is necessary to train the person of the learner and their behavior, above all in interpersonal relationships from a humanistic approach and integral development. For the elaboration of this proposal, the scientific method of direct observation was used in order to identify that cooperative play is often not used by teachers as an alternative educational tool to promote the development of positive values and attitudes in the individual. The method that was used before, during and after the execution of the proposal was the exploratory, descriptive and correlational study variables. In addition, bibliographic documentation was used to support the research and the results obtained. It was concluded that teachers practice very little cooperative games with children, ignoring this pedagogical tool as a strategy to solve conflicts in the classroom in a positive, healthy way and collective activities are carried out sporadically; premium plus individual work than team work.
\end{abstract}

1 Universidad Técnica de Ambato, Facultad de Ciencias de la Salud, Ambato, Ecuador, r.andrade@uta.edu.ec
2 Universidad Técnica de Ambato, Facultad de Ciencias de la Salud, Ambato, Ecuador,
noemiandradealban@gmail.com
3 Universidad Técnica de Ambato, Facultad de Ciencias de la Salud Ambato, Ecuador, vi.penafiel@uta.edu.ec 
Keywords: Cooperative play, strategy, values, positive attitudes, education, school environment.

\section{Resumen.}

Las expresiones lúdicas no solamente han beneficiado al hombre en su historia, si bien actualmente se sabe que es una actividad clave para la formación del hombre en relación con los demás, con la naturaleza y consigo mismo en la medida en que le propicia un equilibrio estético y moral entre su interioridad y el medio con el que interactúa. El objetivo de este estudio es desarrollar una propuesta educativa que impulse el desarrollo de actitudes positivas de los niños tanto en el aula como fuera de ella, a través de la ejecución de los juegos cooperativos que permita promover de manera directa el ambiente escolar de los niños de Educación Inicial. En esta línea es preciso formar la persona del educando y su comportamiento, ante todo en las relaciones interpersonales desde un enfoque humanístico y de desarrollo integral. Para la elaboración de esta propuesta se utilizó el método científico de la observación directa con el fin de identificar que el juego cooperativo a menudo no es utilizado por las docentes como herramienta educativa alternativa para impulsar el desarrollo de valore y actitudes positivas en el individuo. El método que se utilizó antes, durante y después de la ejecución de la propuesta fue el exploratorio, descriptivo y correlacional de las variables de estudio. Además, se utilizó documentación bibliográfica que sustentan la investigación y los resultados obtenidos. Se concluyó que las docentes practican muy poco los juegos cooperativos con los niños, desconociendo esta herramienta pedagógica como estrategia para solucionar conflictos en el aula de manera positivas, saludable $\mathrm{y}$ las actividades colectivas son realizadas esporádicamente; prima más el trabajo individual que el trabajo en equipo

Palabras claves: Juego cooperativo, estrategia, valores, actitudes positivas, educación, ambiente escolar.

\section{Introducción.}

El juego es la forma más humana de ser libres, de crear nuevas historias, comprender al otro y convivir en armonía; según (Linaza, 2013, p.2), "Afirmar que el juego es una necesidad es reconocer la forma específica con la que los niños abordan la realidad, sea física, social o intelectual" para (Fujimoto, 2014, Vol.3). "El juego es universal y constituye un elemento clave en el desarrollo infantil. Es el mecanismo que contribuye al desarrollo motriz, cognitivo, afectivo, social." Por otra parte, Bijou citado en (Galiano, 2014), define el juego "como una forma específica que tienen los niños de relacionarse con otros seres humanos y con el mundo en general" Aceptando la tesis anterior (Linaza \& Jurgo Torres, 2014), afirman que "El hábito entra en la vida como juego. La actividad lúdica se le considera una actividad 
indispensable para el desarrollo personal. Se la recomienda como actividad placentera y formativa."

Las características que posee el juego citado por (Gómez, 2016), siguiendo a (García López, Gutiérrez Hidalgo, \& Otros, 2000), son las siguientes: Placentero: produce placer a los jugadores, natural y motivador ya que es espontaneo, voluntario no hay obligación, sino que es una actividad libre, Mundo aparte somete al participante en un mundo paralelo y de ficción, donde él puede lograr metas que no es capaz de conseguir en el mundo real, una de las características del juego es que es creador y favorece la creatividad al generar escenarios fantasiosos. Expresivo a través del juego ponemos de manifiesto sentimientos, comportamientos y es un ente socializador ya que se da la interacción con los demás. También favorece aspectos como la cooperación, la convivencia y el trabajo en equipo.

Debido a la importancia que estos autores le dan al juego en la vida del niño fue desarrolla y ejecutada una propuesta que ayuda tanto al niño como a los docentes a generar ambientes escolares saludables y al desarrollo de actitudes positivas tomando como herramienta el juego cooperativo. "Si al juego lo dotamos de un componente didáctico, observamos que es una gran estrategia metodológica, ya que acerca al alumno al aprendizaje de forma activa y la aleja de lo meramente memorístico" (Chacón, 2008). Según (Orlick, 1990), define el juego cooperativo como un conjunto, libertades que contribuyen al "Desarrollo de la cooperación, de los buenos sentimientos y del apoyo mutuo." Para (Marina, 2013)“los juegos cooperativos son propuestas que buscan disminuir las manifestaciones de agresividad en los juegos promoviendo actitudes de sensibilización, cooperación, comunicación y solidaridad" y requieren la participación de todos para conseguir un objetivo común, esta idea es también compartida por otros autores como (Galiano, 2014) quien considera que "la cooperación y el trabajo en equipo son herramientas fundamentales en todo ser humano. Su desarrollo, formación e incorporación en el día a día del centro escolar y desde edades tempranas, hace que este valor adquiera gran importancia" el autor (Hernandez M. , 2014), hace referencia a estos juegos como un medio "que, aplicado con rigurosidad y constancia, permite y facilita procesos de autoconocimiento, interacción, socialización, e inclusión, en la comunidad no solo escolar sino deportiva."

\section{Características de los juegos cooperativos}

(Rutz, 2013), en su escrito toma las características de estos juegos citado por los autores más relevantes del juego cooperativo Omeñaca y Ruiz (1999), quienes manifiestan que una de las características de los juegos cooperativos es que es una Actividad conjunta y participativa, que necesita que todos los participantes colaboren entre ellos, además de esta otra característica importante es que, Exige la coordinación de tareas, para la consecución de un fin común que Permite explorar y desarrollar la creatividad en la búsqueda de soluciones. Favorece las relaciones "empáticas, cordiales y constructivas" (1999, p.46) entre los compañeros. Da más importancia al proceso que al resultado final, posibilita el aprendizaje 
de valores morales y de destrezas de carácter social. (Pinto, Girón, \& Villegas, 2015). No existe la competición, es decir, no hay rivales y por lo tanto no se enfrentan entre los participantes. No excluye ni discrimina: todas las personas aportan algo al grupo, y por ultimo una de las características es que No elimina, lo que permite seguir experimentando y, por ende, tener una mayor experiencia.

\section{Actitudes positivas y valores para una convivencia saludable}

El concepto de valor abarca muchos significados y contenidos diferentes desde un punto de vista socio educativo, para (Bertussi, 2014), los valores son un conjunto de elementos que contribuyen a ordenar y reproducir históricamente la vida y las relaciones sociales, es en donde radica la importancia de los valore. Además según algunos autores son actitudes que se adquieren siendo modificables y educables, (Ocáriz, Lavega, Mateu, \& Rovir, 2014), la autora (Garaigordobil, 2014) también afirma el enunciado anterior en su artículos los juegos cooperativos.

Cuando pensamos en la escuela o ámbitos educativos nos ponemos a pensar que valores se deben tratar (Ocáriz, Lavega, Mateu, \& Rovir, 2014), refieren a los valores que son compartidos por todos que hacen referencia a la Declaración Universal de los Derechos Humanos que son: libertad, respeto, dialogo, cooperación, igualdad. (Jaqueira \& 0tros, 2014), establece el termino Educación Física para la Paz para determinar que "aspectos de la Educación para la Paz pueden ser trabajados desde la Educación Física.” No se trata de abarcar todo desde esta área, sino de aprovechar este ámbito para tratar elementos y situaciones que se producen en ella.

Para poner énfasis en la importancia de las actitudes positivas, y la capacidad que tiene el ser humano para socializar tomare en cuenta los siguientes conceptos, (Lacunza, 2012,p.1-20). Habilidades sociales descritas como un "conjunto de estrategias que permiten interactuar de forma constructiva en los diferentes ámbitos de relación social de la persona". Abarca desde componentes verbales hasta los no verbales: gestos, saludos, dialogar, la mirada y la prosocialidad que se refiere a "conductas de carácter interpersonal entre cuyos fines esta beneficiar de algún modo al otro." Los juegos cooperativos son una herramienta que permite trabajar actitudes positivas y valores en los niños de una manera divertida con igualdad de condiciones para todos los participantes, según los autores ya antes mencionados (Ocáriz, Lavega, Mateu, \& Rovir, 2014). establecen tres ámbitos donde se desarrollan varios de estos "valores universales": en primer lugar, valores para la convivencia y la vida en equilibrio con el entorno (libertad, responsabilidad, tolerancia, dialogo, respeto, amistad, cooperación, solidaridad y paz), en segundo lugar, valores para el bienestar personal (alegría, autoestima y autosuperación) y en tercer lugar, valores relacionados con las capacidades unidas a la propia actividad física (competencia motriz, creatividad motriz y salud). 
Según (Marina, 2013) quien expresa que el juego cooperativo puede desarrollar las siguientes actitudes: capacidades para poder resolver problemas, sensibilidad para reconocer como se siente el otro, sensibilidad para aprender a valorar y expresar la importancia del otro y la capacidad para poder expresar sentimientos, emociones, problemas. El autor (Rios, Vallejo Gerena, Niño Camacho, \& García Arbeláez, 2016), indica que el juego cooperativo puede permitir el desarrollo de valores y actitudes como la empatía, dialogo, confianza, autoestima y la alegría.

También a (Garaigordobil, 2014), en la entrevista que le realiza Iñigo Marauri (2009), se le plantea la siguiente pregunta: "con juegos más cooperativos y menos competitivos, ¿se promoverán unos comportamientos adultos más cooperativos y menos competitivos?" a lo que ella no duda en responder "sin ninguna duda, los juegos contiene y enseñan valores." En su artículo competencia emocionales como predictores de conductas pro sociales la autora (Ruvalcaba Romero, 2017) expone que existen ·Diversas contribuciones científicas han demostrado las importantes relaciones existentes entre las competencias socioemocionales y aspectos específicos en el ámbito escolar." (Mora \& Basten, 2013), afirman que "La actividad lúdica en los niños es una de los principales métodos estratégicos que construyen y contribuyen al aprendizaje, mediante su aplicación se puede lograr manifestar y desarrollar valores que serán útiles para toda la vida.” En su escrito (Ramirez, 2015), dice lo siguiente "Los primeros años de vida en el ser humano son de vital importancia para la adquisición de habilidades que necesitara en la edad adulta, es por eso que la etapa de educación inicial debe y requiere ser estimulada en todos los sentidos."

\section{Ambiente Escolar para un desarrollo integral}

Se tomó en cuenta la importancia del ambiente escolar en los niños de educación inicial, ya que es el comienzo de su vida educativa, y de las experiencias de convivencia y las relaciones interpersonales que ellos tengan impactara de manera positiva o negativa su paso por la vida educativa. Algunos autores consideran el ambiente escolar como "la interrelación entre los diferentes miembros de un establecimiento educativo, que tiene incidencia significativa en el desarrollo ético, socio afectivo e intelectual de los estudiantes." Afirmado por (Mendoza, Ballestero, \& Cortés, 2014), citado por (Banz, 2008, p.3), para (Pérez \& Ramírez, 2015), se refiere a condiciones adecuadas para que se lleve a cabo un fin último el aprendizaje y más aún un aprendizaje significativo, son una combinación de elementos internos como las relaciones interpersonales dentro de la escuela y externos a la institución ya sean aspectos administrativos como el edificio escolar y el espacio de aula. Ciado por (Rinaldi, 2009), plantea que las vivencias y experiencias en el aula dejan huellas y memorias en el ambiente, por lo tanto, resulta indispensable evaluar, elegir los materiales, el espacio y el mobiliario.

(Olivos, 2014), considera que existen dos tipos de ambientes el primero hace referencia al espacio físico, como iluminación, espacio, clima, ventilación, colores y el segundo hace referencia a las sensaciones y sentimientos que la persona experimenta con las personas que 
en el habitan; para la psicología ambiental se llama ambiente emocional. El autor (García \& Soler, 2015, p.151-161), afirma que el docente también cumple un papel muy importante en la educación de valores y actitudes positivas en el niño.

Partiendo de lo antes mencionado, el ambiente escolar no solo involucra el factor humano si no también el factor físico es decir "todo aquello que rodea al ser humano, lo que influye en su vida y como el influye en su entorno" por lo tanto en el ambiente escolar se interrelacionan elementos sociales, físicos, emocionales, culturales psicológicos y actitudinales. (Manriquez, 2014). De la misma manera opina (Caldwell, 1993), quien enfatiza en que el ambiente está compuesto por factores relacionados entre sí como, lo físico, lo social, lo humano y lo histórico.

(Gutiérrez-Méndez \& Pérez-Archundia, 2015) En su artículo de estrategias para mejorar la convivencia escolar afirma que "Educar para la paz y la convivencia reconoce una visión de la realidad susceptible de ser transformada, no es estable ni definitiva, sino cambiante y provisional, y que, en consecuencia, se puede construir otro tipo de relaciones sociales." El autor (Abad, 2006, p.1), insiste en que se debe contar con "un espacio educativo que exprese y comunique el proyecto pedagógico, como un compromiso de participación del entorno sociocultural al que pertenece" en donde se busca la conexión entre el espacio físico o infraestructura y el proyecto pedagógico de las personas inmersas en él, que sea un ambiente educativo en donde se prepare al niño para la vida en donde se pueda explorar, demostrar afecto, crear nuevas experiencias, que se ofrezca tiempo y espacio para jugar y explorar el entorno.

El objetivo general de estudio es proponer la práctica del juego cooperativo como herramienta didáctica y determinar su eficacia para estimular y desarrollar actitudes positivas que promuevan el mejoramiento del ambiente escolar de los niños de Educación Inicial, tomando como referencia lo expuesto por (Ocáriz, Lavega, Mateu, \& Rovir, 2014). Los objetivos específicos serán favorecer la aparición de conductas cooperativas, como la empatía, descubrir la importancia del otro, la demostración de emociones y el trabajo en equipo. Tomando en cuenta lo expuesto por el autor (Banz, 2008, p.3), se observará a los niños de Educación Inicial en su ambiente escolar antes, durante y después de la ejecución de la propuesta basado en las características de los juegos cooperativos que favorecen las relaciones interpersonales y ayudan a mejorar el ambiente escolar.

\section{Metodología}

Para la elaboración de este artículo se contó con la suficiente información bibliográfica que sustentan teóricamente las bases del documento, se obtuvo información relevante sobre los juegos cooperativos y como estos favorecen las relaciones interpersonales de los niños, de inicial, se recopilo información sobre las actitudes positivas y valores que desarrollan los juegos cooperativos y la importancia de un ambiente escolar integral. Se obtuvo información 
de libros, documentos digitales, artículos científicos, páginas web, revistas, videos que permitieron la sustentación teórica de este artículo. Según (Ferrari, 2010, p.78) es la revisión bibliográfica del tema para conocer el estado de la cuestión. La búsqueda, recopilación, organización, valoración, crítica e información bibliográfica sobre un tema específico, tiene un valor pues evita la dispersión de publicaciones o permite la visión panorámica de un problema."

La investigación fue de campo tomando en cuenta lo expuesto por (Colón, 2007,p.87), al enunciar lo siguiente "En este capítulo se establece el análisis del trabajo de campo, ordenamiento que supone un reporte estructurado de los hallazgos empíricos, encaminado todo a revelar los observables que den respuestas a las interrogantes de investigación y a los objetivos planteados." Realizada en la Unidad Educativa "Francisco Flor" de la ciudad de Ambato, Ecuador, con la participación de 52 estudiantes de Educación Inicial con un rango de edad de 3 a 4 años, se utilizó la ficha de observación directa, lo cual permitió observar a los niños antes, durante y después de la ejecución de la propuesta, se entrevistó a las docentes de cada paralelo por separados, para obtener los resultados se observó a los niños en el aula, al momento de salir al recreo, cuando realizaban sus tareas diarias, en la hora del lunch, al ejecutar los juegos y actividades varias.

Al referirse a los espacios utilizados para la realización de la propuesta, fue amplio se utilizó las aulas de clases y el patio de descanso, los materiales utilizados fueron proporcionados por la autora de esta propuesta, todos los niños tuvieron el material necesario para realizar cada actividad propuesta se ha utilizado globos, pelotas, telas, sillas, música entre otros materiales

Por otro lado, se trabajó con cada grupo de niños por separado, con la presencia de las docentes responsables cada grupo o paralelo constaba de 26 niños en donde se encontraron algunas observaciones como problemas de comportamiento, el no saber respetar el turno de cada persona, el trabajo en equipo era muy escaso, había niños que no participaban de los juegos y se observó agresiones físicas entre los niños. Todas las ejecuciones de los juegos estuvieron basados en la cooperación e integración de cada uno de los miembros de los equipo con señalan algunos autores, (Lacunza, 2012,p.1-20), (Jaqueira \& 0tros, 2014).

Una vez recogida la información, fue organizada y clasificada de cada grupo cabe enfatizar que con ambos grupos utilice los mismos materiales, el mismo tiempo y los mismos ítems para recogerla información necesaria sobre la aparición y demostraciones de conductas cooperativas, participación activa de cada participante y apariciones de conductas poco sociables

La ficha de observación fue analizada, corregida y validada por docentes de la Universidad Técnica de Ambato. Se obtuvo los datos necesarios para realizar el análisis e interpretación dela ficha de observación, con el fin de obtener resultados que colaboren con los objetivos de la propuesta. Para realizar la comprobación de la hipótesis de investigación se sustrajo 4 
preguntas significativas con el fin de obtener un resultado claro que ayude en el entendimiento de los objetivos de la indagación de información.

De acuerdo con nivel de significancia $\alpha=0,05$ y con 6 grados de libertad, se obtuvo como valor del Chi cuadrado calculado $\mathrm{x}^{2} \mathrm{c}=29.03$ siendo mayor que el valor del Chi cuadrado tabular $x_{t}^{2}=12,591$, en donde se rechaza la hipótesis nula y es aceptada la hipótesis alterna teniendo como conclusión que, Los juegos cooperativos SI desarrollan actitudes y valores positivos que mejoran el ambiente escolar de los niños de educación inicial.

\section{Resultado de las encuestas}

Tabla 1: Frecuencias Observadas

\begin{tabular}{|c|c|c|c|c|}
\hline Ítems & Siempre & A veces & Nunca & Total \\
\hline $\begin{array}{llll}\text { Pregunta } 1 . & \text { Interacción } & \text { entre } \\
\text { compañeros } & & & \end{array}$ & 30 & 14 & 8 & 52 \\
\hline Pregunta 2 Resolución de conflictos & 40 & 10 & 2 & 52 \\
\hline Pregunta 3. Búsqueda de apoyo & 26 & 19 & 7 & 52 \\
\hline Pregunta 4 Participación activa & 48 & 4 & 0 & 52 \\
\hline Total & 144 & 47 & 17 & 208 \\
\hline
\end{tabular}

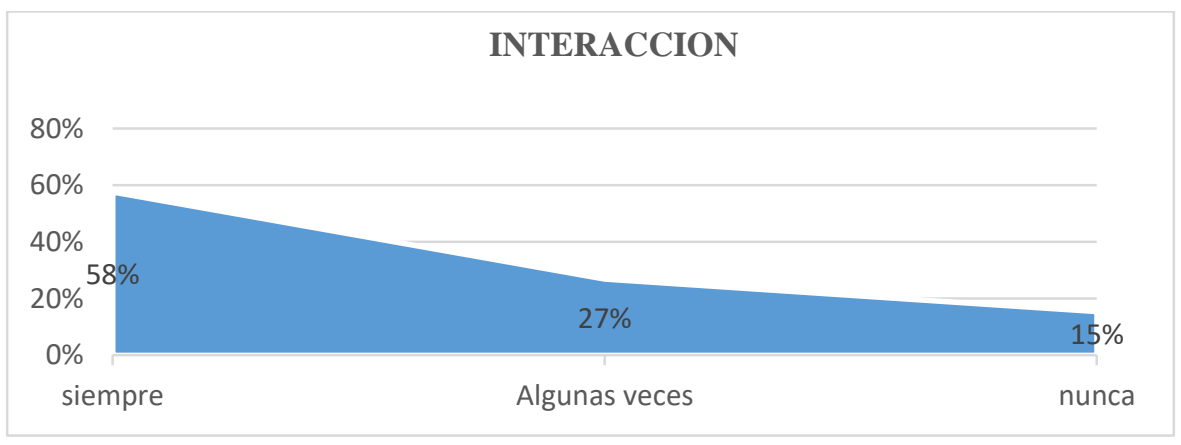

Figura 1: Resultado ficha de observación

Resultados observados de acuerdo a la frecuencia en la que los niños de educación inicial interactuaban con sus compañeros antes de la ejecución de los juegos cooperativos. 


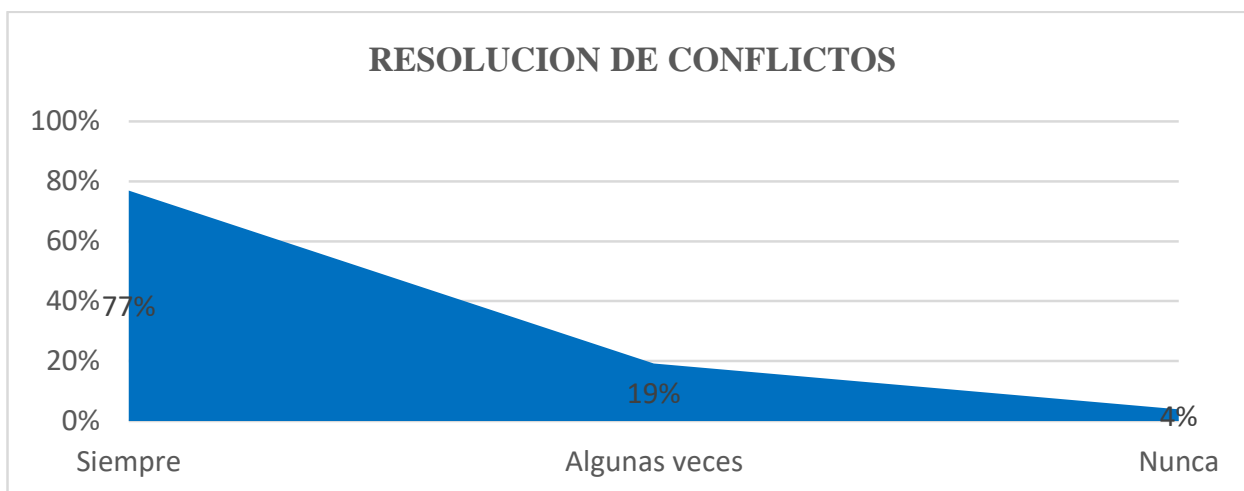

Figura 2: Resultado Ficha de observación

Resultados observados de acuerdo a la resolución de conflictos que presentaban los estudiantes de educación inicial a sus compañeros durante la ejecución de la propuesta de los juegos cooperativos.

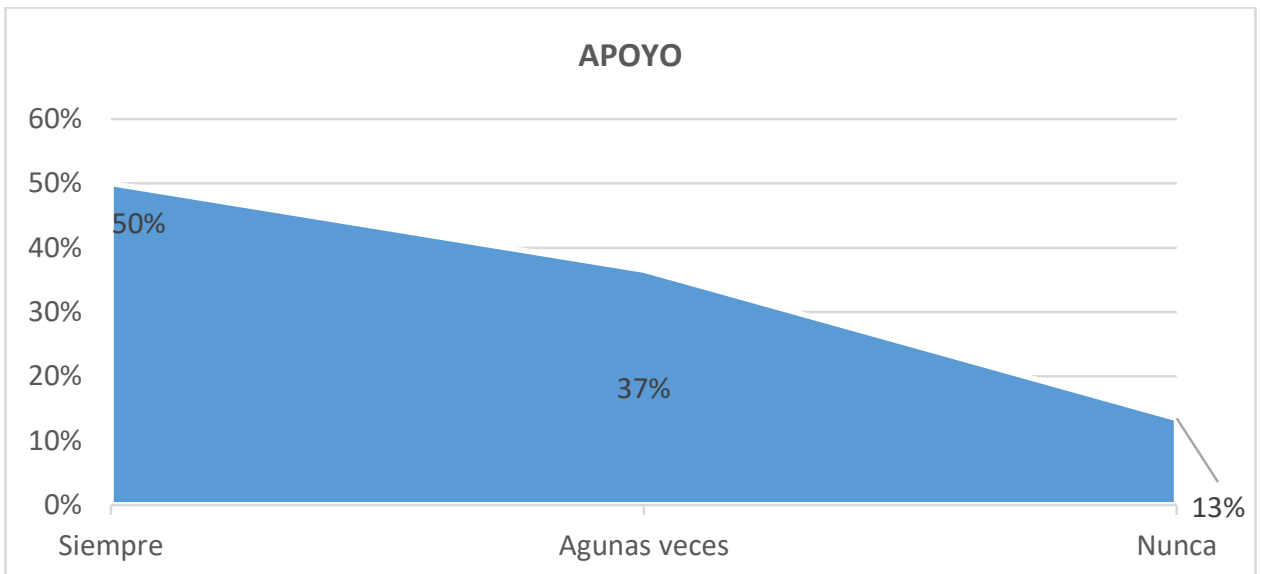

Figura 3: Resultado ficha de observación

Resultados observados de acuerdo a la frecuencia en la que los niños de educación inicial solicitaban apoyo a sus compañeros antes de la ejecución de los juegos cooperativos.

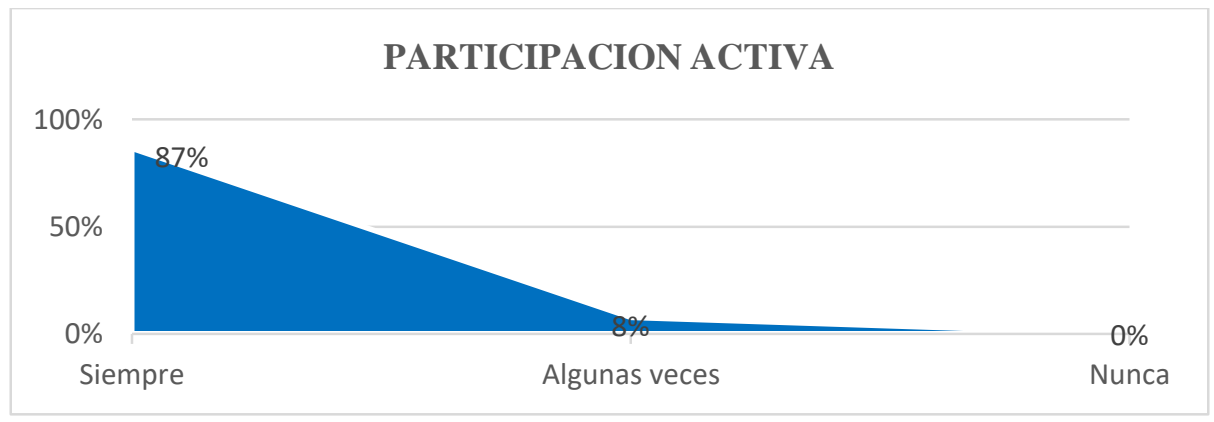

Figura 4: Resultado Ficha de observación 
Resultados observados de acuerdo a la participación activa que se observó en los estudiantes de educación inicial a sus compañeros durante la ejecución de la propuesta de los juegos cooperativos.

\section{Resultado de las encuestas}

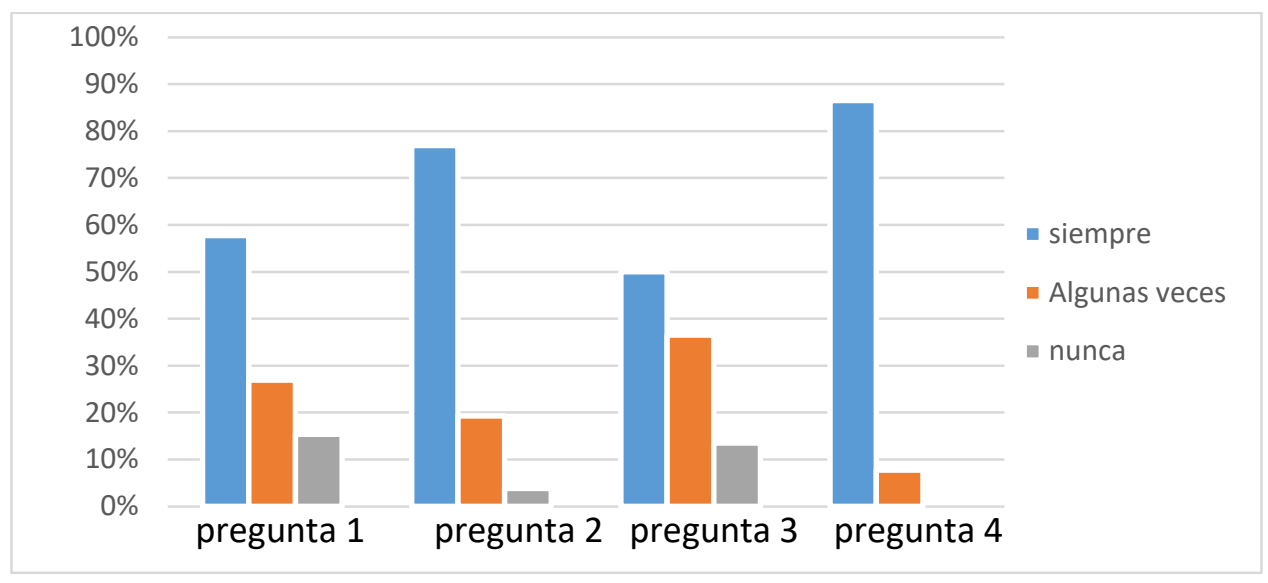

Figura 3: Resultado general sobre la ficha de observación de los juegos cooperativos

Desarrollo del análisis de la ficha de observación establecida para conocer si el juego cooperativo permite desarrollar actitudes y valores positivos para el mejoramiento del ambiente escolar de los niños de educación inicial.

\section{Comprobación de Hipótesis}

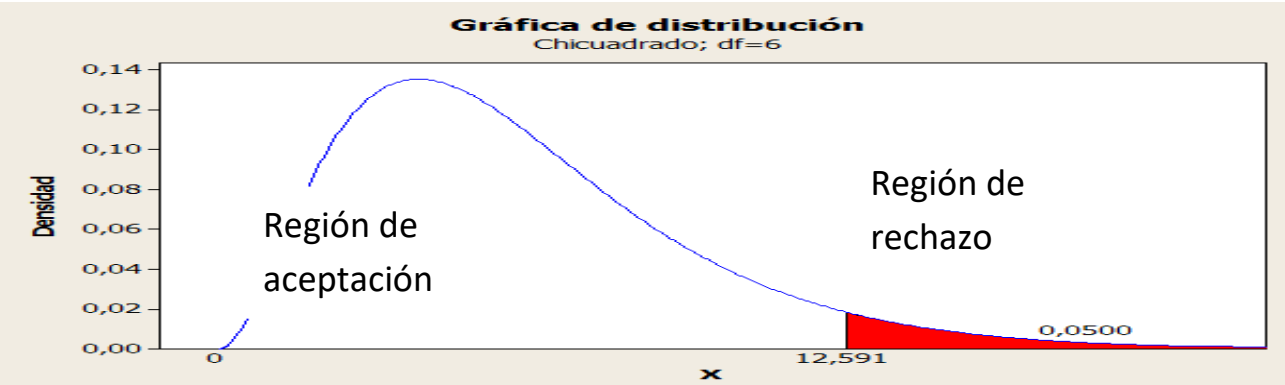

Figura 4: Comprobación de la hipótesis

$\mathrm{t}=12.592$

$X^{2} c=29,3$

2.2 Formulas

$X^{2}=\sum\left(0-E^{2}\right)$ 
En donde:

$\mathrm{X}^{2}$ Chi- Cuadrado

$\sum=$ Sumatoria

$\mathrm{O}=$ Frecuencia Observada

$\mathrm{E}=$ Frecuencia esperada

Niveles significativos y Reglas de Decisión

Grados de Libertad

Para establecer los grados de libertad se utiliza la siguiente formula:

$\mathrm{gl}=(\mathrm{c}-1)(\mathrm{f}-\mathrm{l})$

$\mathrm{gl}=(3-1)(4-1)$

$\mathrm{gl}=2 * 3$

$\mathrm{gl}=6$

\section{Grados de significación}

$\infty_{-} 0.05 \quad \mathrm{X}^{2} \mathrm{t}=12.592$

El valor de $\mathrm{x} 2$ con 6 grados de libertad y un nivel de significancia o límite de tolerancia de 0.05 y X2t de 12.592(valor de la tabla de puntos de distribución X2)

\section{Resultados}

Se trabajó con 52 estudiantes de la Unidad Educativa "Francisco Flor" de la ciudad de Ambato - Ecuador, se observó a los niños en dos grupos, se interactúo con ellos con los mismos materiales y se aplicó el mismo tiempo de ejecución de los juegos, para obtener la información necesaria se exploró con una ficha de observación, dicho instrumento esta direccionado para identificar las dificultades que presentan los niños en su ambiente escolar e identificar si los juegos cooperativos permiten el desarrollo de las actitudes y valores positivos y el mejoramiento del ambiente escolar.

A través de los resultados obtenidos se puede decir que la interacción de los niños antes de la ejecución de los juegos cooperativos se observó en porcentajes altos 30 niños de los 52 observados interactuó con sus compañeros a la hora de clases y de descanso, 14 niños de los 52 observados interactuó algunas veces con sus compañeros, a su vez se observó que 8 niños nunca interactuaron con sus compañeros, en presencia del docente varios de ellos conversaban, se prestaban los materiales, reían y en ocasiones conversaban de los juguetes 
que tenían en casa y que habían hecho en sus casa. Los niños que no interactuaban con sus compañeros en clases ni fuera de ella jugaban solos y no conversaban con sus compañeros permanecían callados y realizaban la actividad propuesta por la docente en silencio y en ocasiones presentaban conflictos con sus compañeros al no querer compartir las cosas con sus compañeros, algunos de estos niños jugaban con niños de otro paralelo y no con sus compañeros.

Durante la ejecución del juego cooperativo, se presentaban obstáculos propios del juego como pasar por debajo de una silla mantener la pelota dentro de una tela sin dejar que esta caiga, no dejar que el globo se caiga entre otros. Un porcentaje de 40 niños ayudaban a sus compañeros y superaban los obstáculos y buscaban como hacerlo en equipo, guiaban a los demás para hacer el trabajo, al inicio cierto grupo de niños (10) no resolvían los problemas con prontitud y se observaba la frustración y no querían seguir en el juego, en ese instante se explicaba de nuevo el juego para hacer que todos participaran sin inconvenientes 2 niños no permitían que el desarrollo del juego avance en la primera y segunda sección de juegos, con ellos se trabajó más para permitir la participación de todos en los juegos.

A través de los resultados de la ejecución de la propuesta la búsqueda de ayuda por parte de los niños observados fue constante antes de la ejecución de la propuesta del juego cooperativo 26 niños buscaban el apoyo y la ayuda constantemente en sus compañeros y la docente, al momento de realizar una actividad como pintar dibujar, realizar manualidades solicitaban ayuda y los demás respondían positivamente ante la necesidad de los compañeros. Algunas veces 19 delos 52 solicitaban ayuda a sus compañeros eran un poco independiente y hacían sus cosas solos, no así 7 de los 52 niños observados que nunca solicitaban apoyo a sus compañeros aun así necesitándola la docente sabia preguntar porque es necesario solicitar ayuda cuando no se entiende cómo hacer algo y lo importante que es el brindar ayuda a quien lo solicita.

El juego permite la participación activa de los niños y fomenta la comunicación en el grupo y la interacción en un mayor porcentaje después de la ejecución de la propuesta se puede decir que 48 niños de los 52 observados participa activamente en clases, colabora con sus compañeros, hace preguntas a la docente si no entiende una actividad, interactúan mucho más que antes de la ejecución de la propuesta, se pudo observar que 2 niños que antes no interactuaban de manera constante ahora lo hacen, en el grupo se logra observar compañerismo y aceptación.

Durante la ejecución de la propuesta se observó que para un mejoramiento del ambiente escolar es necesario que los estudiantes sepan saber participar, es trascendental que los estudiantes participen del medio que los rodea activamente e interactúen con los demás elementos que integran el ambiente, el saber cooperar, propiciar ambientes cooperativos y no competitivos ni individualista contribuye a la mejora del ambiente escolar; ser tolerantes desde las edades tempranas, es necesario educar a los estudiantes en tolerancia hacia los 
demás y respetar las diferencias. Disponer de habilidades sociales, las adquisiciones de habilidades sociales básicas son necesarias para gestionar positivamente las relaciones interpersonales siendo un aspecto básico para mejorar el ambiente escolar; ser solidarios aspecto fundamental que intervienen de manera positiva en el ambiente escolar con factores prosociales. Gestionar pacíficamente conflictos interpersonales, la transformación constructiva de los conflictos en herramientas y habilidades sociales para su desarrollo en los centros educativos es un pilar fundamental para aprender a convivir.

\section{Discusión}

Los resultados señalan que antes de la ejecución de los juegos cooperativos la interacción entre los niños era buenas, pero aún existía parte de ellos que no interactuaban de manera constante, después de la ejecución de los juegos cooperativos se pudo disminuir la brecha y la interacción y la participación activa aumento de manera significativa. Estos resultados confirman los estudios de (Cuestas Cañadas \& Otros, 2016), quien afirma que los beneficios de la utilización de los juegos cooperativos es múltiple, no solo favorecen a la participación activa si no también desarrollan en los niños valores, auto aceptación algunos grupos de estudios han confirmado el efecto positivo del aprendizaje cooperativo para estimular una mejora del clima del aula, aceptando la observación de (Cuéllar de Lucas , Pérez-Brunicardi, , \& De la Iglesia, 2015), quien afirma estas conclusiones al decir que el juego en sí mismo es un medio ideal para la integración, además afirma que los estudios realizados sobre el juego cooperativo y los beneficios en el desarrollo infantil concluyen que este tipo de juegos promueve conductas positivas como el compartir.

Se evidencia de forma constante que la práctica de juegos cooperativos si mejora de forma considerable el ambiente escolar por medio de las actitudes y valores positivos que estos fomentan coincidiendo con los estudios de (Martínez, R, Cepero, M, Collado, D, \& Padial, , 2014), en donde aseguran que los juegos cooperativos ayudan a relacionarse de manera asertiva a los estudiantes con sus compañeros. Esto confirma lo que certifica (Fujimoto, 2014, Vol.3), en su estudio indicando que es trascendental para el desarrollo integral delos niños el juego, la recreación, las artes, la cultura que benefician de manera directa su salud emocional y su desarrollo social. Durante la intervención con los juegos cooperativos se pudo observar que los niños buscaban a sus compañeros para realizar las actividades, se prestaban ayuda entre sí, mostraban empatía hacia sus compañeros y brindaban ayuda si era de (Diaz, 2014), quien necesario estos resultados afirman los estudios realizados por (Garaigordobil, 2014), quien afirma que el juego cooperativo estimula la comunicación, la cooperación con los iguales y el desarrollo de variadas habilidades sociales.

Algunos estudios han relacionado la práctica de actividad física y juegos con la mejora de los estados de ánimo como es el caso afirma que los estudiantes al inicio de cada sección no mostraban cambios en su estado de ánimo, este cambio se observaba al final de las sesiones en donde los estudiantes dejaban de estar tensos y mostraban una actitud más relajada. En 
desacuerdo con las tesis anteriores expone la autora (Rutz, 2013) "La mayoría de los juegos cooperativos tienen un carácter competitivo, los juegos transportan la idea de la competencia que se ejecutan bajo condiciones sencillas y dentro de los límites de la norma".

\section{Conclusiones}

- Se pudo observar que las docentes practican muy poco los juegos cooperativos con los niños, desconociendo esta herramienta pedagógica como estrategia para solucionar conflictos en el aula de manera positivas, saludable y las actividades colectivas son realizadas esporádicamente; prima más el trabajo individual que el trabajo en equipo.

- Es necesario la ejecución constante de esta propuesta tomando en cuenta lo expresado por (Hernandez M. , 2014), para observar cambios de actitud y comportamientos en los niños así como para desarrollar y poner en práctica las habilidades que brinda estos tipos de juego, aceptando el concepto sobe el juego como un estilo de vida en los niños, es la manera más directa de aprender habilidades para la vida, como valores y actitudes que le permitirán un mejor bienestar emocional y social.

- Al establecer los juegos cooperativos como herramienta para la adquisición y desarrollo de actitudes y valores positivos para el mejoramiento del ambiente escolar, se pone énfasis en la importancia de la vida escolar inicial de los niños y en el derecho que tienen ellos a la recreación y al juego, (Fujimoto, 2014, Vol.3), ya que de la manera en la cual ellos experimenten, viven y se relacionen con su entorno en etapas tempranas, marcarán su vida educativa de manera positiva o negativa.

- Para obtener resultados positivos y mejora, el ambiente escolar de los niños es primordial transmitir valores y conocimientos que mejoren su vida y desarrollen en el actitud de compañerismo, colaboración, respeto, tolerancia, empatía, apoyo, cooperación, aceptación al otro, que mejore su autoestima y estimule la ayuda al prójimo y el respeto a las diferencias personales, (Ocáriz, Lavega, Mateu, \& Rovir, 2014).

\section{Referencias Bibliográficas.}

Bertussi. (2014). Los valores y la Moral en la Escuela. Obtenido de https://scholar.go.es/scholar?start=10\&q=los+valores+morales+en+los+ni\%C3\%B1 os\&hl=es\&as_sdt=0,5\&as_ylo=2013\&as_yhi=2018

Cuéllar de Lucas, Pérez-Brunicardi, D., \& De la Iglesia, M. (13 de Julio de 2015). ENSEÑANZA COOPERATIVA COMO INSTRUMENTO PARA LA INCLUSIÓN DE UN NIÑO CON TRASTORNO DE ESPECTRO AUTISTA (TEA) MEDIANTE JUEGOS COOPERATIVOS EN UN AULA DE EDUCACION IN 
Eertussi. (2014). Los valores y la Moral en la Escuela. Obtenido de https://scholar.google.es/scholar?start=10\&q=los+valores+morales+en+los+ni\%C3 $\%$ B1os\&hl=es\&as_sdt=0,5\&as_ylo=2013\&as_yhi=2018

Calderón , D. (2016). El autoetima y el aprendizaje en las matemáticas de los estudiantes de octavo año de Educación General B'asica de la Unidad Educativa Teniente Hugo Ortiz . Ambato : Universidad Técnica de Ambato .

Cartagena, M. (2008). Relación entre la autoeficacia en el Rendimiento Escolary los Hábitos de estudio en el rendimiento Académico en alumnos de Secundaria. Revista Iberoamericana sobre Calidad, Eficacia y Cambio en Educación, 65.

Cuéllar de Lucas , Y., Pérez-Brunicardi, , D., \& De la Iglesia, M. (13 de Julio de 2015). ENSEÑANZA COOPERATIVA COMO INSTRUMENTO PARA LA INCLUSIÓN DE UN NIÑO CON TRASTORNO DE ESPECTRO AUTISTA (TEA) MEDIANTE JUEGOS COOPERATIVOS EN UN AULA DE EDUCACION INFANTIL. Obtenido de http://www.grupocieg.org/archivos_revista/Ed.\%2021\%20(259271)\%20Cu\%C3\%A91lar\%20de\%20Lucas\%20y\%20otros\%20$\% 20$ septiembre\%202015_articulo_id213.pdf

Cuestas Cañadas, C., \& Otros. (21 de Abril de 2016). La Contribución de los Juegos Cooperativos a la Mejora Psicomotriz en Niños de Educación Infantil. Recuperado el 14 de Marzo de 2018, de La Contribución de los Juegos Cooperativos a la Mejora Psicomotriz en Niños de Infantil: https://ruidera.uclm.es/xmlui/handle/10578/13797

Diaz, M. (2014). LOS JUEGOS COOPERATIVOS Y SU INCIDENCIA EN LOS ESTADOS DE ÁNIMO Y LAS EMOCIONES EN ESCOLARES DE 10-12 AÑOS - Obtenido de Revista Digital de Educacion Fisica: https://dialnet.unirioja.es/servlet/articulo?codigo=5558017

Fernandez , N., \& García , M. (2007). El Aprendizaje Cooperativo como estrategia de Enseñanza-Aprendizaje en Psicopedagogía (UC):repercusiones y valoraciones de los estudiantes. Revista Iberoamaricana de educación, 02.

Fujimoto, G. (5 de Abril de 2014, Vol.3). El derecho del niño al juego, a las artes y a las actividades recreativas. Revista Latinoamericana de Educación Infantil, 113-124. Obtenido de El derecho del niño al juego, las artes y las actividades recreativas: http://redaberta.usc.es/reladei

G, M. R. (1976). Anto- control: Nociones basicas e investigación funadamental . Revista Latinamericana de Psicologia , 390 . 
Galiano, O. (2014). Juegos y Actividades Cooperativas. Recuperado el enero de 2018, de http://academica-e.unavarra.es/handle/2454/13987

Garaigordobil, M. (23 de Mayo de 2014). El juego cooperativo. Obtenido de www.researchgate.net:

https://www.researchgate.net/publication/31838923_El_juego_cooperativo_para_pr evenir_la_violencia_en_los_centros_escolares_M_Garaigordobil_Landazabal_JM_ Fagoaga_Azumendi

García, G., \& Soler, E. S. (2015, p.151-161). Revista Cubana de Reumatología. Obtenido de La disciplina y el aprendizaje como valores en la actividad docente: http://scielo.sld.cu/scielo.php?script=sci_arttext\&pid=S1817$59962015000300013 \& \operatorname{lng}=$ es\&tlng=pt.

Gómez, A. (2016). RETOS COOPERATIVOS FÍSICOS EN EDUCACIÓN INFANTIL . Recuperado el 12 de Marzo de 2018, de http://uvadoc.uva.es/handle/10324/18391: http://uvadoc.uva.es/handle/10324/18391

Gutiérrez-Méndez, D., \& Pérez-Archundia, E. (Eneo-Junio de 2015). ESTRATEGIAS PARA GENERAR LA CONVIVENCIA ESCOLAR . Revista Ra Ximhai, 11(1), 63 81. Obtenido de http://www.redalyc.org/pdf/461/46139401004.pdf

Hernandez , C., Rodrígues , N., \& Vargas ,Á. (2012). Los Hábitos de estudio y la motivación para el aprendizaje de los alumnos de tres carreras de ingenieria . México : Instituto de Eduación Superior México .

Hernandez, M. (Junio de 2014). JUEGOS COOPERATIVOS: UNA HERRAMIENTA PARA AFIANZAR EL TRABAJO EN EQUIPO . Obtenido de http://repository.unilibre.edu.co/handle/10901/7649

Jaqueira, A., \& 0tros. (1 de Marzo de 2014). Educando para la paz jugando: género y emociones . Obtenido de Educando para la paz jugando: género y emociones: http://revistas.um.es/educatio/article/view/194071

Lacunza, A. B. (13 de Marzo de 2012,p.1-20). Obtenido de Revista Pequén 2012, Escuela de Psicología, Universidad del Bío-Bío: http://hdl.handle.net/10596/2490

Linaza. (28 de Febrero de 2013, p.2). Goole academico. Obtenido de https://repositorio.uam.es/handle/10486/662399

Linaza, \& Jurgo Torres, S. (2014). El juego en el Desarrollo Infaltil. Obtenido de https://www.researchgate.net/publication/268265700_El_juego_en_el_desarrollo_in fantil 
Manriquez, S. (Diciembre de 2014). Convivencia y clima escolar: claves de la gestión del conocimiento. Ultima Decada; Proyecto Juventudes, 22(41), 153-178. Recuperado el 12 de Marzo de 2018, de https://scielo.conicyt.cl/scielo.php?pid=S0718$22362014000200007 \&$ script=sci_arttext\&tlng=en

Marina, J. (2013). Valores y Virtudes. Obtenido de Brujula para Educadores: http://www.pediatriaintegral.es/wp-content/uploads/2013/xvii03/07/222224\%20Brujula\%203.pdf

Martínez, R, Cepero, M, Collado, D, \& Padial, . (2014). ADQUISICIÓN DE VALORES Y ACTITUDES MEDIANTE EL JUEGO Y EL DEPORTE EN EDUCACIÓN FÍSICA, EN EDUCACIÓN SECUNDARIA. Obtenido de Google academico: http://www.journalshr.com/papers/Vol\%206_N\%203/V06_3_3.pdf

Mendoza, H., Ballestero, R., \& Cortés. (20 de Agosto de 2014). El Clima Escolar Como Elemento Fundamental de la Convivencia en la Escuela . Escenario, 12(2), 7-18. Recuperado el 12 de Marzo de 2018, de http://ojs.uac.edu.co/index.php/escenarios/article/view/311

Mora, V., \& Basten, M. (Septiembre-Diciembre de 2013). Aplicación de la propuesta para fortalecer la vivencia de los valores de solidaridad y empatía con niños y niñas de 4 a 5 años, de una institución privada de Heredia, por medio de talleres lúdicoscreativos. (M. Vargas Mora, \& M. Basten, Edits.) Revista Electronica Educare, 17(3), 199-228. Recuperado el 12 de Marzo de 2018, de URL: http://www.una.ac.cr/educare

Ocáriz, S. d., Lavega, Mateu, \& Rovir. (2014). EMOCIONES POSITIVAS Y EDUCACIÓN DE LA CONVIVENCIA ESCOLAR. CONTRIBUCIÓN DE LA EXPRESIÓN MOTRIZ COOPERATIVA. Revista de Investigación Educativa, 32(2), 309-326. Obtenido de Revista de Investigacion Educativa: http://revistas.um.es/rie/article/view/183911/163191

Olivos, P. (16 de Mayo de 2014). Ambientes Escolares. Recuperado el 13 de Marzo de 2018, de Articulos Academicos: https://www.researchgate.net/publication/236172095_Ambientes_Escolares

Peñarrieta , L. (2010). Desarrollo de habilidades comunicativas básicas en niños de 9 a 13 años a traves de la radio.Comuni@cción, 53.

Pérez, C., \& Ramírez, M. (Septiembre-Diciembre de 2015). Los ambientes de aula que promueven el aprendizaje, desde la perspectiva de los niños y niñas escolares. Revista Digital Educare, 19(3), 1-32. doi:http://dx.doi.org/10.15359/ree.19-3.11 
Pinto, Girón, \& Villegas, M.-L. (2 de Febrero de 2015). LOS VALORES DE LA FAMILIA EN LA EDUCACIÓN INICIAL. Revista Multidisciplinaria Dialogica, 5-27. Obtenido de LOS VALORES DE LA FAMILIA EN LA EDUCACIÓN INICIAL: https://dialnet.unirioja.es/servlet/articulo?codigo=5237738

Ramirez, C. (2015). IDEAD PROGRAMA DE LICENCIATURA EN PEDAGOGÍA INFANTIL IBAGUÉ 2015. Recuperado el 12 de Marzo de 2018, de http://repository.ut.edu.co/handle/001/154

Rios, L. F., Vallejo Gerena, S., Niño Camacho, L., \& García Arbeláez, J. (18 de Junio de 2016). Revista Iberoamericana. Obtenido de SOCIOAFECTIVIDAD Y DESARROLLO MORAL EN LA PRIMERA INFANCIA: http://revistas.iberoamericana.edu.co/index.php/rhpedagogicos/article/view/903/837

Rutz, P. (2013). El juego cooperativo como estrategia . Obtenido de https://prezi.com/4mzmpmajqbja: http://200.23.113.51/pdf/29636.pdf

Ruvalcaba Romero, G. G. (2017). Competencias socioemocionales como predictoras de conductas prosociales y clima escolar positivo en adolescentes. Revista interuniversitaria de formación del profesorado, 77-90. Recuperado el 12 de Marzo de 2018, de https://dialnet.unirioja.es/servlet/articulo?codigo=5980962

FANTIL. Obtenido de enseñanza cooperativa como instrumento para la inclusión de un niño con trastorno de espectro autista (tea) mediante juegos cooperativos en un aula de educacion

INFANTIL: http://www.grupocieg.org/archivos_revista/Ed.\%2021\%20(259271)\%20Cu\%C3\%A91lar\%20de\%20Lucas\%20y\%20otros\%20$\% 20$ septiembre\%202015_articulo_id213.pdf

Diaz, M. (2014). Los juegos cooperativos y su incidencia en los estados de ánimo y las emociones en escolares de 10-12 años . Obtenido de Revista Digital de Educacion Fisica: https://dialnet.unirioja.es/servlet/articulo?codigo=5558017

Fernandez , N., \& García , M. (2007). El Aprendizaje Cooperativo como estrategia de Enseñanza-Aprendizaje en Psicopedagogía (UC): repercusiones y valoraciones de los estudiantes. Revista Iberoamaricana de educación, 02.

Fujimoto, G. (5 de abril de 2014, Vol.3). El derecho del niño al juego, a las artes y a las actividades recreativas. Revista Latinoamericana de Educación Infantil, 113-124. Obtenido de El derecho del niño al juego, las artes y las actividades recreativas: http://redaberta.usc.es/reladei 
Galiano, O. (2014). Juegos y actividades cooperativas como elemento positivo en el desarrollo infantil. Recuperado el enero de 2018, de juegos y actividades cooperativas como elemento positivo en el desarrollo infantil: http://academicae.unavarra.es/handle/2454/13987

Garaigordobil, M. (23 de mayo de 2014). El juego cooperativo para prevenir la violencia en los centros escolares. Obtenido de www.researchgate.net/publication/31838923

García, G., \& Soler, E. S. (2015, p.151-161). Revista Cubana de Reumatología. Obtenido de La disciplina y el aprendizaje como valores en la actividad docente: http://scielo.sld.cu/scielo.php?script=sci_arttext\&pid=S1817$59962015000300013 \& \operatorname{lng}=$ es\&tlng=pt.

Gómez, A. (2016). RETOS COOPERATIVOS FÍSICOS EN EDUCACIÓN INFANTIL. Recuperado el 12 de Marzo de 2018, de http://uvadoc.uva.es/handle/10324/18391: http://uvadoc.uva.es/handle/10324/18391

Gutiérrez-Méndez, D., \& Pérez-Archundia, E. (Eneo-Junio de 2015). ESTRATEGIAS PARA GENERAR LA CONVIVENCIA ESCOLAR . Revista Ra Ximhai, 11(1), 63 81. Obtenido de http://www.redalyc.org/pdf/461/46139401004.pdf

Hernandez, M. (Junio de 2014). JUEGOS COOPERATIVOS: UNA HERRAMIENTA PARA AFIANZAR EL TRABAJO EN EQUIPO DE LA CATEGORÍA JUVENIL DE LA SELECCIÓN DE CUNDINAMARCA FEMENINA DE VOLEIBOL. Obtenido de http://repository.unilibre.edu.co/handle/10901/7649

Jaqueira, A., \& Otros. (1 de Marzo de 2014). Educando para la paz jugando: género y emociones en la práctica de juegos cooperativos competitivos . Obtenido de Educando para la paz jugando: género y emociones en la práctica de juegos cooperativos competitivos : http://revistas.um.es/educatio/article/view/194071

Lacunza, A. B. (13 de Marzo de 2012,p.1-20). LAS HABILIDADES SOCIALES Y EL COMPORTAMIENTO PROSOCIAL INFANTIL DESDE LA PSICOLOGÍA POSITIVA1. Obtenido de Revista Pequén 2012, Escuela de Psicología, Vol. 2, n 1 , p. 1 - 20. Universidad del Bío-Bío: http://hdl.handle.net/10596/2490

Linaza. (28 de Febrero de 2013, p.2). Goole academico. Obtenido de EL JUEGO ES UN DERECHO Y UNA NECESIDAD DE LA INFANCIA: https://repositorio.uam.es/handle/10486/662399

Linaza, \& Jurgo Torres, S. (2014). El juego en el Desarrollo Infaltil. Obtenido de https://www.researchgate.net/publication/268265700_El_juego_en_el_desarrollo_in fantil 
Manriquez, S. (Diciembre de 2014). Convivencia y clima escolar: claves de la gestión del conocimiento. Ultima Decada; Proyecto Juventudes, 22(41), 153-178. Recuperado el 12 de Marzo de 2018, de https://scielo.conicyt.cl/scielo.php?pid=S0718$22362014000200007 \&$ script=sci_arttext\&tlng=en

Marina, J. (2013). Valores y Virtudes. Obtenido de Brujula para Educadores: http://www.pediatriaintegral.es/wp-content/uploads/2013/xvii03/07/222224\%20Brujula\%203.pdf

Martínez, R, Cepero, M, Collado, D, \& Padial, . (2014). ADQUISICIÓN DE VALORES Y ACTITUDES MEDIANTE EL JUEGO Y EL DEPORTE EN EDUCACIÓN FÍSICA, EN EDUCACIÓN SECUNDARIA. Obtenido de Google academico: http://www.journalshr.com/papers/Vol\%206_N\%203/V06_3_3.pdf

Mendoza, H., Ballestero, R., \& Cortés. (20 de Agosto de 2014). El Clima Escolar Como Elemento Fundamental de la Convivencia en la Escuela. Escenario, 12(2), 7-18. $\begin{array}{lllll}\text { Recuperado el } 12 \text { de } & \text { Marzo de }\end{array}$ http://ojs.uac.edu.co/index.php/escenarios/article/view/311

Mora, V., \& Basten, M. (Septiembre-Diciembre de 2013). Aplicación de la propuesta para fortalecer la vivencia de los valores de solidaridad y empatía con niños y niñas de 4 a 5 años, de una institución privada de Heredia, por medio de talleres lúdicoscreativos. (M. Vargas Mora, \& M. Basten, Edits.) Revista Electronica Educare, 17(3), 199-228. Recuperado el 12 de Marzo de 2018, de URL: http://www.una.ac.cr/educare

Ocáriz, S. d., Lavega, Mateu, \& Rovir. (2014). EMOCIONES POSITIVAS Y EDUCACIÓN DE LA CONVIVENCIA ESCOLAR. CONTRIBUCIÓN DE LA EXPRESIÓN MOTRIZ COOPERATIVA. Revista de Investigación Educativa, 32(2), 309-326. Obtenido de http://revistas.um.es/rie/article/view/183911/163191

Olivos, P. (16 de Mayo de 2014). Ambientes Escolares. Recuperado el 13 de Marzo de 2018, de Artículos Académicos: https://www.researchgate.net/publication/236172095_Ambientes_Escolares

Pérez, C., \& Ramírez, M. (Septiembre-Diciembre de 2015). Los ambientes de aula que promueven el aprendizaje, desde la perspectiva de los niños y niñas escolares. Revista Digital Educare, 19(3), 1-32. doi: http://dx.doi.org/10.15359/ree.19-3.11

Pinto, Girón, \& Villegas, M.-L. (2 de Febrero de 2015). LOS VALORES DE LA FAMILIA EN LA EDUCACIÓN INICIAL. Revista Multidisciplinaria Dialógica, 5-27. Obtenido de LOS VALORES DE LA FAMILIA EN LA EDUCACIÓN INICIAL: https://dialnet.unirioja.es/servlet/articulo?codigo $=5237738$ 
Rios, L. F., Vallejo Gerena, S., Niño Camacho, L., \& García Arbeláez, J. (18 de Junio de 2016). Revista Iberoamericana. Obtenido de SOCIOAFECTIVIDAD Y DESARROLLO MORAL EN LA PRIMERA INFANCIA: http://revistas.iberoamericana.edu.co/index.php/rhpedagogicos/article/view/903/837

Rutz, P. (2013). El juego cooperativo como estrategia para mejorar las relaciones interpersonales y disminuir la agresividad en niños de segundo de preescolar. Obtenido de https://prezi.com/4mzmpmajqbja/los-juegos-cooperativos-comoestrategia-didactica-para-mejor/: http://200.23.113.51/pdf/29636.pdf

Ruvalcaba Romero, G. G. (2017). Competencias socioemocionales como predictoras de conductas prosociales y clima escolar positivo en adolescentes. Revista interuniversitaria de formación del profesorado, 77-90. Recuperado el 12 de Marzo de 2018, de https://dialnet.unirioja.es/servlet/articulo?codigo=5980962

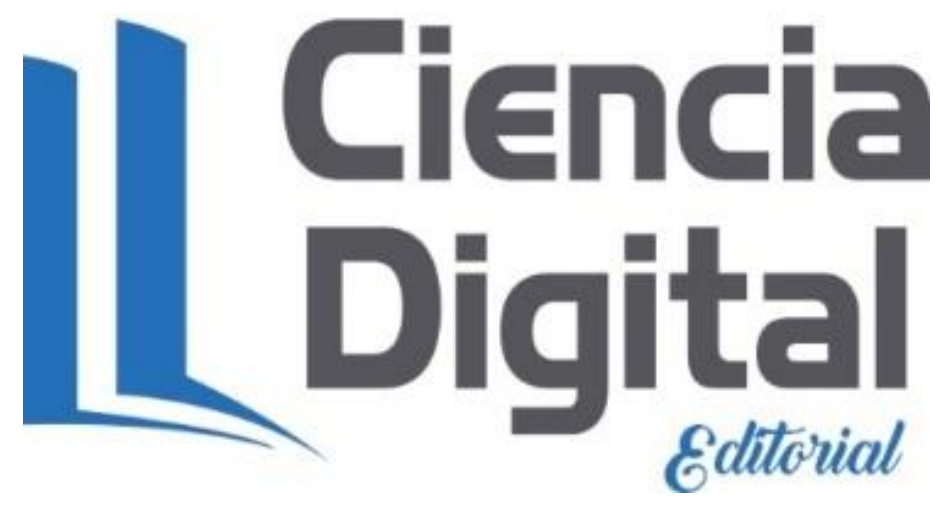




\section{Para citar el artículo indexado.}

Andrade J., Andrada N. \& Peñafiel V. (2019). Estrategia psicopedagógica en la educación. Revista electrónica Ciencia Digital 3(1), 217-238. Recuperado desde: http://cienciadigital.org/revistacienciadigital2/index.php/CienciaDigital/article/view/285/68 $\underline{2}$

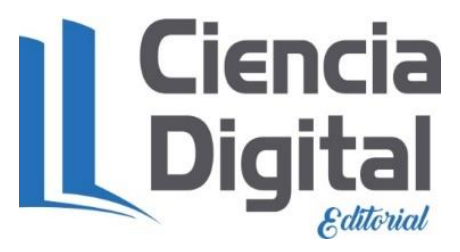

El artículo que se publica es de exclusiva responsabilidad de los autores y no necesariamente reflejan el pensamiento de la Revista Ciencia Digital.

El artículo queda en propiedad de la revista y, por tanto, su publicación parcial y/o total en otro medio tiene que ser autorizado por el director de la Revista Ciencia Digital.
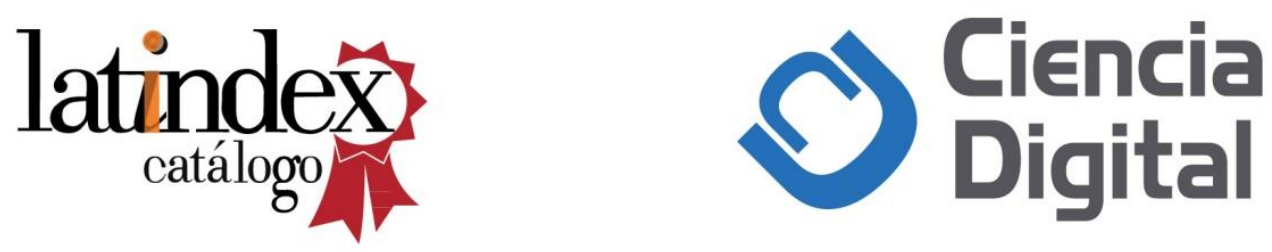